\title{
Surgical Management of Undescended Testis in Infants, Single Scrotal Incision Orchiopexy Versus Traditional Inguinal Approach: A Comparative Study
}

\author{
Omar Atef Elekiabi ${ }^{1, ~ *, ~ E h a b ~ M a h r o o s ~ O r a b y ~}{ }^{2}$, Mohamed Abdallah Zaitoun ${ }^{3}$, \\ Abdelwahab Saleh Almoregy \\ ${ }^{1}$ Department of Pediatric Surgery, Faculty of Medicine, Zagazig University, Zagazig, Egypt \\ ${ }^{2}$ Department of General Surgery, Faculty of Medicine, Benha University, Benha, Egypt \\ ${ }^{3}$ Department of General Surgery, Faculty of Medicine, Zagazig University, Zagazig, Egypt
}

Email address:

Omar_ekiabi@gmail.com (O. A. Elekiabi)

${ }^{*}$ Corresponding author

\section{To cite this article:}

Omar Atef Elekiabi, Ehab Mahroos Oraby, Mohamed Abdallah Zaitoun, Abdelwahab Saleh Almoregy. Surgical Management of Undescended Testis in Infants, Single Scrotal Incision Orchiopexy Versus Traditional Inguinal Approach: A Comparative Study. Journal of Surgery. Vol. 8, No. 5, 2020, pp. 158-162. doi: 10.11648/j.js.20200805.13

Received: June 13, 2020; Accepted: June 22, 2020; Published: September 3, 2020

\begin{abstract}
Background: Surgical intervention is mostly needed in many cases of undescended testis to improve fertility; it is still unsure which approach is better than the other in management of undescended testicle in infants; classic inguinal approach or scrotal single incision approach. Aim of the current study was to compare between classic inguinal approach and scrotal single incision approach to perform orchiopexy for management of un-descended testis in infants and to clarify which is better; inguinal orchidopexy or scrotal orchidopexy. Patients and methods: we included forty male patients with undescended testis who were divided into two groups; group A included twenty patients and they were surgically managed with single transscrotal orchiopexy and group B included twenty patients and they were surgically managed with classic inguinal approach by two incisions (inguinal and scrotal). We assessed operative time and complications as wound infection, recurrence and testicular atrophy. Results: Operative time in group A was shorter than the operative time of group B which was managed by the inguinal approach $(\mathrm{p}=0.004)$. Immediate post-operative complications as; skin site infection, hematoma and edema, in group A were slightly more than the complications in group $B(p=0.002)$. group A showed no recurrence or testicular atrophy after six months while group B showed 2 cases of recurrence and one case of testicular atrophy $(p=0.005)$. At three months after surgery, cosmetic results and patients satisfaction of the incision scar was higher in group A than group B ( $<0.001)$. Conclusions: performing orchiopexy through single scrotal incision approach is better than the approach of inguinal incision regarding operative time, rabid healing time, parents' satisfaction, fewer long-term complications, less incidence of testicular atrophy and recurrence.
\end{abstract}

Keywords: Undescended Testis, Single Scrotal Incision, Inguinal Approach

\section{Introduction}

Undescended testis is considered a developmental anomaly and its prevalence ranged from $2-4 \%$ at birth to about $1 \%$ after the first year of age [1]. The exact reason of its occurrence is unknown by it has many risk factors as; lack of sufficient gonadotropin hormones stimulation, exogenous estrogen administration by the mother, gubernaculums and epididymal anomalies in addition to many abnormalities of the abdominal wall [2]. Majority of cases of undescended testes descends in the $1^{\text {st }}$ few months of infants' life and few of them descend in the next 9 months [3]. Treatment by exogenous hormones is a treatment option which gives about $30 \%$ therapeutic response [4]. Surgical intervention is mostly needed in many cases to improve fertility [5].

Inguinal approach is considered the traditional surgical treatment procedure of undescended testicle and it included 
two incisions which are the inguinal incision to expose the inguinal canal and the scrotal incision to perform orchiopexy [6]. The inguinal incision was suggested to be more helpful in spermatic cord and hernia sac exposure and mobilization in addition to allowing high sac ligation for achieving a sufficient length for the testis to be positioned and fixed in the scrotum [7, 8]. Performing the approach of a single scrotal incision was primarily described by in 1989 by Bianchi and Squire [9], as a new approach which might reduce morbidities occurred after the classic inguinal approach. After that many authors stated that scrotal orchidopexy might be defective in reaching sufficient length of the spermatic cord for high position of the testis, other researchers stated that such approach could not allow a sufficient patent processus vaginalis high ligation for prevention of inguinal hernia [10]. So it is still unsure which approach is better than the other in management of undescended testicle in infants; classic inguinal approach or scrotal single incision approach.

Aim of the current study was to compare between classic inguinal approach and scrotal single incision approach to perform orchiopexy for management of undecended testis in infants and to clarify which is better; inguinal orchidopexy or scrotal orchidopexy (SO).

\subsection{Patients and Methods}

Current study is a prospective randomized one which included forty male patients with undescended testis who underwent undergoing surgery in Pediatric Surgery Department, Zagazig University Hospital, and General Surgery Department, Benha University, between April 2019 and May 2020, were recruited.

\subsection{Criteria of Patients' Inclusion in the Study}

We included; male patients less than two years old who were diagnosed with undescended testis with palpable testis in the inguinal canal.

\subsection{Criteria of Patients' Exclusion from the Study}

We excluded patients with previous history of abdominal and/or inguinal surgery, ectopic testis, ambiguous genitalia, hypogonadism, hormonal disorders, retractile testis, patients with inter abdominal testis or with their testis located above the inguinal canal.

The present study was approved by the institutional review board of Faculty of Medicine, Benha University and Zagazig University and we obtained a written informed consent from parents of the patients or their official guardians. We examined all included children in a supine position two times, before and after general anesthesia induction for confirmation of the diagnosis of undescended testis and its exact location. We measure the base line size of the testis once the re-measure it during the period of follow-up.

The included forty patients were divided into two groups, where group A included twenty patients and they were surgically managed with single trans-scrotal orchiopexy and group B included twenty patients and they were surgically managed with classic inguinal approach by two incisions (inguinal and scrotal).

\subsection{Surgical Procedures}

\subsubsection{Surgical Management of Patients in Group A}

We made an incision in the skin and subcutaneous tissues of the scrotum and we dissected the fascia to the tunica vaginalis. We divided attachments of gubernaculum. We mobilized the spermatic cord by a gentle traction of the testis. We cut the fibers of cremasteric muscle to facilitate the needed mobilization. We separated the hernia sac from the spermatic cord and then we high-ligated it. In case of needing additional length of the spermatic cord we made a more dissection through opening the canal and the external ring around the cord. We created a dartos pouch then we fixed the testis inside it to the tunica albuginea using chromic suture. Finally, we repaired dartos fascia then the skin.

\subsubsection{Surgical Management of Patients in Group B}

For patients managed with the traditional inguinal approach, we performed two incisions in the skin; the first is the inguinal incision which allowed an access to the inguinal canal for visualization of the structures of the spermatic cord structures for allowing separation the high hernia sac ligation to achieve a sufficient length for positioning the testis in the scrotum. We made a scrotal incision then we created a dartos pouch then we fixed the testis inside it to the tunica albuginea using chromic suture. Finally, we repaired dartos fascia then the skin.

For patients in the two groups we assessed operative time and complications as wound infection and testicular atrophy. We used ultrasonography for detection of testicular size.

Primary surgical outcomes are; operative time and postoperative wound infection and secondry outcomes are recurrence and testicular atrophy.

We followed all patients after discharge after two weeks, three months, six months and a year after surgeries for identification of recurrence, any complications and to determine location and size of testis.

We asked the parents about satisfaction with post-operative cosmetic results and categorized them as satisfied or unsatisfied.

\subsection{Statistical Data Analysis}

Statistical analysis has been using the SPSS version. 17.0 (Chicago, USA). We used the following tests for analysis of collected data from patients; t-test, chi-square test, and fisher's exact test considering the $\mathrm{p}<0.05$ as a statistically significant value.

\section{Results}

We included forty male patients with unilateral or bilateral undescended testis. General data of included patients were found in table 1 .

Twenty patients underwent single scrotal incision (group A) 
and the remaining twenty patients underwent traditional inguinal approach (group B).

Table 1. Associations between Single Scrotal Incision Orchiopexy and Traditional Inguinal approach regarding base line findings.

\begin{tabular}{|c|c|c|c|c|}
\hline \multirow{3}{*}{ Variables } & \multirow{2}{*}{ Total } & \multicolumn{3}{|l|}{ Surgical techniques } \\
\hline & & \multicolumn{2}{|r|}{ Traditional Inguinal approach } & \multirow[b]{2}{*}{$\mathbf{p}$} \\
\hline & $\mathrm{N}=40(\%)$ & $\mathrm{N}=\mathbf{2 0}(\mathbf{5 0} \%)$ & $\mathrm{N}=\mathbf{2 0}(\mathbf{5 0} \%)$ & \\
\hline \multicolumn{5}{|l|}{ Age groups: } \\
\hline At birth & $30(75)$ & $13(65)$ & $17(85)$ & \multirow{4}{*}{0.009} \\
\hline$<1$ years old & $8(20)$ & $6(15)$ & $2(10)$ & \\
\hline$>1$ years old & $2(5)$ & $1(5)$ & $1(5)$ & \\
\hline Weight & $6 \pm 1.5$ & $5.85 \pm 0.53$ & $4.85 \pm 1.53$ & \\
\hline \multicolumn{5}{|c|}{ Comorbid condition: } \\
\hline Absent & $36(90)$ & $18(90)$ & $18(90)$ & \multirow{3}{*}{0.068} \\
\hline Present & $4(10)$ & $2(10)$ & $2(10)$ & \\
\hline \multicolumn{4}{|l|}{ Site: } & \\
\hline Left & $10(25)$ & $2(10)$ & $8(40)$ & \multirow{3}{*}{0.001} \\
\hline Right & $28(70)$ & $17(85)$ & $11(55)$ & \\
\hline Bilateral & $2(5)$ & $1(5)$ & $1(5)$ & \\
\hline
\end{tabular}

Table 2. Associations between Single Scrotal Incision Orchiopexy and Traditional Inguinal approach regarding; operative and postoperative findings.

\begin{tabular}{|c|c|c|c|c|}
\hline \multirow{3}{*}{ Variables } & \multirow{2}{*}{ Total } & \multicolumn{3}{|l|}{ Surgical techniques } \\
\hline & & Single Scrotal Incision & Traditional Inguinal approach & \\
\hline & $\mathrm{N}=40(\%)$ & $\mathrm{N}=20(50 \%)$ & $\mathrm{N}=20(50 \%)$ & $\mathbf{p}$ \\
\hline \multicolumn{5}{|c|}{ Operation time: } \\
\hline Mean \pm SD & $29.33 \pm 15.68$ & $17.07 \pm 10.47$ & $35.6 \pm 12.81$ & 0.004 \\
\hline \multicolumn{5}{|c|}{ Parents satisfaction: } \\
\hline Absent & $3(7.5)$ & $0(0)$ & $3(15)$ & \multirow{2}{*}{$<0.001 * *$} \\
\hline Present & $37(42.5)$ & $20(0)$ & $17(85)$ & \\
\hline \multicolumn{5}{|c|}{ Short term complications: } \\
\hline Absent & $37(42.5)$ & $17(85)$ & $20(100)$ & \multirow{2}{*}{$0.002 *$} \\
\hline Present & $3(7.5)$ & $3(15)$ & $0(0)$ & \\
\hline \multicolumn{5}{|c|}{ Long term complications } \\
\hline No & $36(90)$ & $20(100)$ & $16(80)$ & \multirow{2}{*}{$0.005^{*}$} \\
\hline Yes & $4(10)$ & $0(0)$ & $4(20)$ & \\
\hline
\end{tabular}

\subsection{Demographic and General Results}

Right-sided undescended testis was detected in 28 patients, left-sided undescended testis was detected in 8 and bilateral undescended testis was detected in 4 patients $(p=0.001)$. All included patients were less than 2 years of age. Most cases were presented at birth in both groups and patients managed by single scrotal approach was younger than patients in the other group $(p=0.009)$. No statistically significant results were found between both groups regarding the presence of comorbid conditions.

\subsection{Operative Results}

Operative time in group A which was managed by a single scrotal incision, was shorter than the operative time of the other group which was managed by the inguinal approach (17 \pm 3.84 vs $28 \pm 11 ; \mathrm{p}=0.004)$.

\subsection{Post-operative Results}

No statistically detected differences were found between both groups regarding location of the testis after one week from the operation as most of testes were located in the scrotum.

There were no detected recurrences after one week from the operation in both groups.

Immediate post-operative complications as; skin site infection, hematoma and edema, in group A which was managed by a single scrotal incision, were slightly more than the complications in the other group which was managed by the inguinal approach $(\mathrm{p}=0.002)$. These complications were mild and subsided a week after operation using conservative management and antibiotics.

Follow-up and long term results

group A which was managed by a single scrotal incision showed no recurrence or testicular atrophy after six months while group B which was managed by the inguinal approach showed 2 cases of recurrence and one case of testicular atrophy $(\mathrm{P}=005)$.

We found no statistically significant differences in testicular size at pre-operative baseline measurements and measurements during the period of post-operative follow-up.

At three months after surgery, cosmetic results and patients satisfaction of the incision scar was higher in group A that was managed by a single scrotal incision more than the other group $(\mathrm{p}<0.001)$.

\section{Discussion}

Undescended testis is the commonest discovered 
developmental anomaly in neoborn and it has different incidence [11]. Surgical management is the standard curative management of such disorder to preserve testicular function and prevents its atrophy degeneration [12].

Traditional inguinal approach of orchidopexy that included two incisions was the established management technique for undescended testes. Trans-scrotal approach of orchidopexy that included single incision is a recently proposed management approach [8]. The benefits of the transscrotal approach in management of undescended testis are still under research although it gains a marked popularity [13]. In the present study transscrotal approach is compared with traditional inguinal approach as management approach for infants with undescended testis. We showed that there are many benefits of the transcrotal approach than the inguinal approach due to shorter operative time, fewer complications better recovery in addition to less incidence of recurrence and excellent cosmetic appearance, similarly results of previous studies $[1,5,8]$.

Many previous reports compared both approaches of management of undescended testis and showed that duration of surgery and duration of hospital staying is shorter in orchidopexy using single scrotal-incision than in inguinal approach $[1,7,12]$.

We showed the incidence of complications in the group of patients who were managed by single scrotal incision was slightly more than the other group, similarly Badbarin et al., [1], and $\mathrm{Na}$ et al. [6], showed that incidence of wound infection and hematoma after scrotal incision was more than the classic inguinal technique.

Slightly different results showed by Bassel et al. [14], that complications after single scrotal incision were less than inguinal technique. We showed that there were no recurrence or testicular atrophy in the single trans-scrotal approach similarly results of Badbarin et al., [1] and $\mathrm{Na}$ et al. [6], who showed that there were no reported cases of testicle atrophy were reported after the surgery via scrotal incision technique.

Results of Callewaert et al., [15], were in line with ours that single scrotal incision is a quick and accurate method with fewer complication and excellent cosmetic results.

Zouari et al., [8], study believe that single scrotal incision has plethora of benefits as it is simple, offers little dissection with less trauma to the inguinal region, less postoperative pain and swelling. Moreover, single scrotal incision produced quicker operation, better cosmetic results and higher patients' satisfaction [16].

\section{Conclusions}

Results of our study revealed that performing orchiopexy for management of undescended testis in infants through single scrotal incision approach is better than the approach of inguinal incision regarding operative time, rabid healing time, parents' satisfaction with the cosmetic results in addition of producing fewer long-term complications, less incidence of testicular atrophy and recurrence.

\section{References}

[1] Badbarin D, Ehsan S, Toomatari M, Aslanabadi S, Farhadi E, Salamat SA. A Comparative Study of Single Scrotal Incision Orchiopexy of Children with Palpable Low-Lying Undescended Testis with Traditional Inguinal Method. Adv Pediatr Surg. 2019 Jun; 25 (1): 14-19.

[2] Koivusalo A, Taskinen S, Rintala RJ. Cryptorchidism in boys with congenital abdominal wall defects. Pediatr Surg Int 1998; 13: $143-5$.

[3] Aslan Abadi S, Kazemi Rashed F. Study of prevalence of hidden testis in the children under one year in city of Tabriz with study diagnostic role of the social workers in referral system. Med J Tabriz Univ Med Sci 2006; 28: 25-8.

[4] Esposito C, De Lucia A, Palmieri A, Centonze A, Damiano R, Savanelli A, et al. Comparison of five different hormonal treatment protocols for children with cryptorchidism. Scand J Urol Nephrol 2003; 37: 246-9.

[5] Shaoguang Feng, Huajun Yang, Xiang Li, Junjia Yang, Jie Zhang, Aihe Wang et al., Single scrotal incision orchiopexy versus the inguinal approach in children with palpable undescended testis: a systematic review and meta-analysis Pediatr Surg Int (2016) 32: 989-995.

[6] Na SW, Kim SO, Hwang EC, Oh KJ, Jeong SI, Kang TW, et al. Single scrotal incision orchiopexy for children with palpable low-lying undescended testis: early outcome of a prospective randomized controlled study. Korean J Urol 2011; 52: 637-41.

[7] Karaman I, Karaman A, Erdoğan D, Çavuşoğlu YH. The transscrotal approach for recurrent and iatrogenic undescended testes. Eur J Pediatr Surg 2010; 20: 267-9.

[8] Zouari M, Dhaou MB, Jallouli M, Mhiri R. Single scrotalincision orchidopexy for palpable undescended testis in children. Arab J Urol 2015; 13: 112-5.

[9] Bianchi A, Squire BR. Transscrotal orchidopexy: orchidopexy revised. Pediatr Surg Int 1989; 4: 189-92.

[10] Lais A, Ferro F (1995) Trans-scrotal approach for surgical correction of cryptorchidism and congenital anomalies of the processus vaginalis. Eur Urol 29 (2): 235-238.

[11] Thong M, Lim C, Fatimah H. Undescended testes: incidence in 1,002 consecutive male infants and outcome at 1 year of age. Pediatr Surg Int 1998; 13: 37-41.

[12] Dayanç M, Kibar Y, Tahmaz L, Yildirim I, Peker AF. Scrotal incision orchiopexy for undescended testis. Urology 2004; 64: 1216-8.

[13] Gordon M, Cervellione RM, Morabito A, Bianchi A. 20 years of transscrotal orchidopexy for undescended testis: results and outcomes. J Pediatr Urol 2010; 6: 506-12.

[14] Bassel YS, Scherz HC, Kirsch AJ. Scrotal incision orchiopexy for undescended testes with or without a patent processus vaginalis. J Urol 2007; 177: 1516-8.

[15] Callewaert PR, Rahnama MS, Biallosterski BT, Van Kerrebroeck PE. Scrotal approach to both palpable and impalpable undescended testes: should it become our first choice? Urology 2010; 76: 73-6. 
[16] Ramzan M, Sheikh AH, Qureshi MS, Zubair M, Majid F. Single incision transscrotal versus standard inguino-scrotal orchidopexy in children with palpable undescended testis: our experience from April 2007 to April 2010. Pak J Med Sci 2012; 28: 827-9. 